\title{
Handheld 3D Measuring System Based on DSLR Camera
}

\author{
Urban PAVLOVČIČ, Matija JEZERŠEK, Janez MOŽINA \\ Faculty of Mechanical Engineering, University of Ljubljana, Ljubljana, Slovenia
}

\begin{abstract}
A handheld color 3D measuring system is presented. It consists of a commercial DSLR camera body, a $50 \mathrm{~mm}$ fixed focal length lens and a grating projection system with a built-in camera flash used as a light source. The grating mask is composed of up to 140 equally spaced stripes, with the stripes period of $48 \mu \mathrm{m}$. The projector uses $25 \mathrm{~mm}$ focal length lens. All parts with the exception of the grating projection optics and a ring-shaped connection arm are commercially available.

The 3D shape is reconstructed from a single image using the Fourier Transform Profilometry method. $3 \mathrm{D}$ reconstruction based on the triangulation principle is performed after the phase unwrapping. The measuring system is calibrated using the reference geometry method. The precision of the measuring system is $1.4 \mathrm{~mm}$ for measuring range $700 \times 520 \times 400 \mathrm{~mm}$ at the distance of $2000 \mathrm{~mm}$ and $0.5 \mathrm{~mm}$ for measuring range $300 \times 260 \times 200 \mathrm{~mm}$ at the distance of $1000 \mathrm{~mm}$. The image acquisition time can be as short as $1 / 200 \mathrm{~s}$.

The measuring system is used in various medical research studies in cooperation with the Neurologic Clinic, Department of Dermatovenerology and Department of Traumatology from University Medical Center Ljubljana. In one of the applications the orientation of the human head with respect to the torso is measured to accurately diagnose and to evaluate the course of treatment of patients with torticollis disease. In a second application the skin erosion and wound shape before and after the treatment are monitored. The apparatus has proven to be a robust and easy to use, which is a precondition for efficient, fast and accurate measurements.
\end{abstract}

Keywords: Three-dimensional measurement, surface digitalization, biometrics, profilometry, measurement systems, triangulation, image processing, machine vision.

\section{Introduction}

Optical 3D measuring systems are more and more frequently used in medicine mainly due to their additional depth information compared to photography. Another added value is noncontact, noninvasive and fast measurement, which is especially important in medical applications. The required measuring characteristics of the system depend on the measured part of the human body, required accuracy, size of the surface, measuring environment, personnel operating the system and other circumstances. In some cases, lower accuracy can be overlooked at the expense of the portability, user-friendliness and price.

Many handheld measuring systems are commercially available [1-6]. Their accuracies vary from $0.01-$ $0.5 \mathrm{~mm}$, measuring ranges $0.5-1.2 \mathrm{~m}$ and prices from under $10000 €$ to more than $50000 €$. Most of them use the measuring principle of structured lightning; some of them use lasers as light sources [1, 2, 4-6], others use non-coherent light sources [3, 7]. Our goal was to develop a handheld 3D measuring system for a relatively low price. It should be accurate enough for a specific medical 3D measurement and easy to operate. The DSLR camera was chosen due to its high quality/price ratio. There were some similar attempts in the past [7, 8], but none of them used a built-in flash light as the light source. In [7] authors use an LCD projector to project the stripe pattern and in [8] a laser raster generator, which creates laser lines by deflecting the laser beam with two mirrors. We believe that our solution simplifies the system and reduces the necessity of custom made parts and consequently the price.

\section{Construction}

The apparatus is divided into three main parts (see Fig. 1):1) DSLR camera, 2) its lens and 3) grating projection system. Technical properties of the Nikon D90 DSLR camera are listed in Table 1. 
Table 1. Technical specification of the DSLR camera Nikon D90.

\begin{tabular}{|l|l|}
\hline Camera & Nikon D90 DSLR camera \\
\hline Image sensor & $23.6 \times 15.8 \mathrm{~mm}$ \\
\hline Pixel & $12.3 \mathrm{MP}$ (effective) \\
\hline Sensor type & CMOS \\
\hline Frame size & $4288 \times 2848$ \\
\hline Lens mount & $\mathrm{F}-$ mount \\
\hline ISO sensitivity & $200-3200(+6400$ with boost) \\
\hline Crop factor & 1.5 \\
\hline Flash range & $17 \mathrm{~m}($ at ISO 100$)$ \\
\hline Dimensions & $132 \times 103 \times 77 \mathrm{~mm}$ \\
\hline Weight & $703 \mathrm{~g}$ \\
\hline
\end{tabular}

The camera lens is a Nikkor with a fixed focal length $50 \mathrm{~mm} f / 1.8 \mathrm{G}$ AF-S. Both the camera and the lens are commercially available. The grating projection system (GrPS) is composed of an industrial C-mount lens ( $\mathrm{f}=25 \mathrm{~mm}$, image size: $\left.2 / \mathrm{3}^{\prime \prime}\right)$, a grating mask and a connection arm. A built-in camera flash (4) is used as a GrPS light source. The grating mask is placed on the back side of the holder between the projector lens and the flash lamp. It is custom made by a laser based decoating process of the chromium film on a glass substrate. An LPKF DPL 8 AiVo laser was used with laser power $10 \mathrm{~W}$, pulse frequency $50 \mathrm{kHz}$ and laser beam translation speed $400 \mathrm{~mm} / \mathrm{min}$. We are using two masks with different resolution. Both are rectangular, approximately $8.8 \times 6.6 \mathrm{~mm}$ in size. The first one is composed of 140 equally spaced stripes with $48 \mu \mathrm{m}$ period, where the measuring range is $300 \times 260 \times 200 \mathrm{~mm}$. And the second one has 67 stripes with $97 \mu \mathrm{m}$ period, where the measuring range is $700 \times 520 \times 400 \mathrm{~mm}$.

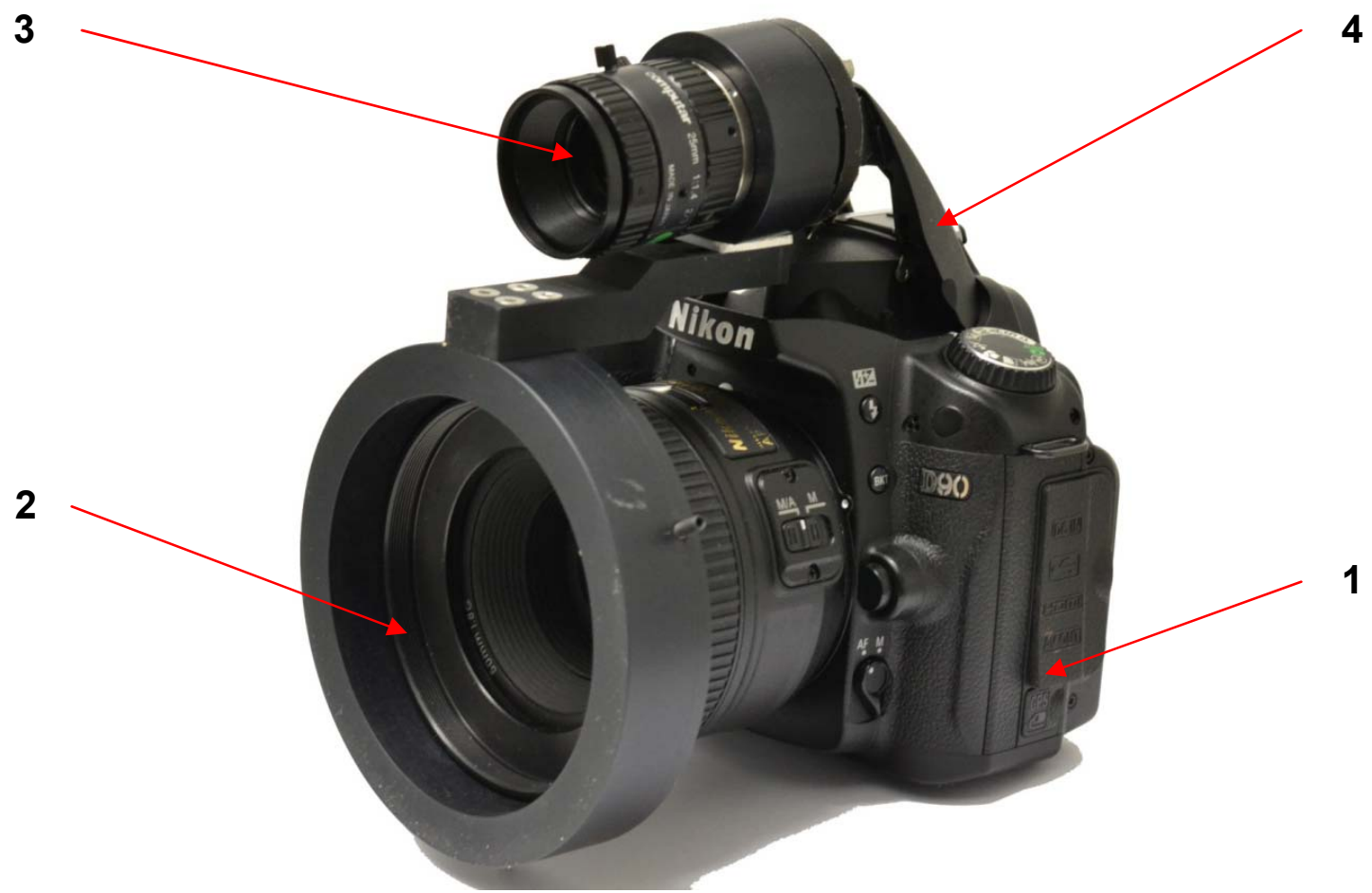

Fig.1. Image of the measuring system. 1) DSLR camera Nikon D90. 2) $50 \mathrm{~mm}$ fixed focal lens. 3) Grating projection system. 4) Built-in flash.

\section{Surface reconstruction}

The 3D surface reconstruction is based on the Fourier transform profilometry (FTP) [9]. It is one of the non-contact 3D measurement methods, where an image of sinusoidal or Ronchi grating, projected on a diffusely reflecting three dimensional object is captured. The surface shape can be calculated from the light pattern (i.e. fringes) deformation. One of the advantages of the method is that it requires only one image of the deformed fringe pattern [12]. 


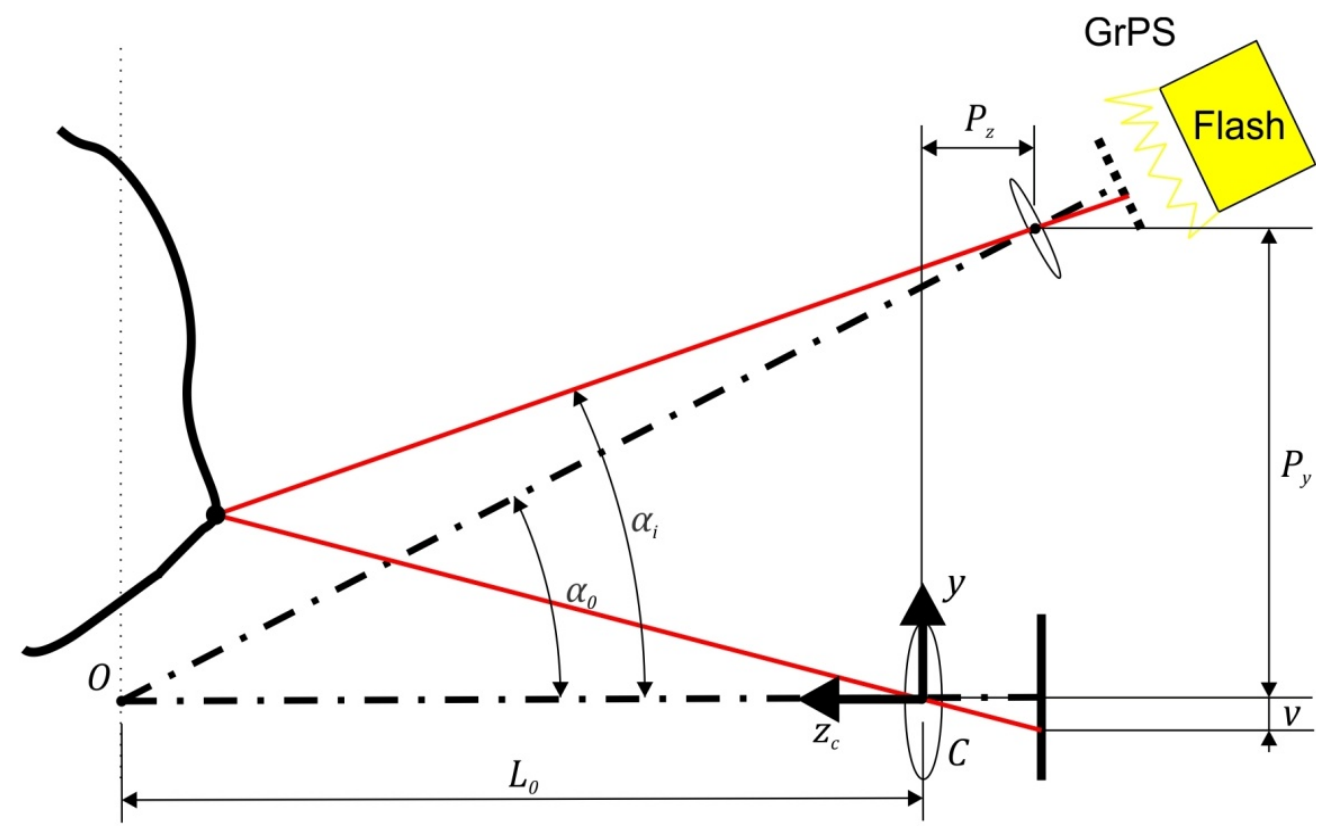

Fig. 2.Optical geometry of the presented system.

The optical geometry is shown in Fig. 2. The measured surface is located on the left side. In the upper right corner is GrPS (flash light and a grating mask in front of it). The vertical distance between the camera and the GrPS lens is $\mathbb{P}_{y}$ and the horizontal $P_{z}$. The intersection of the optical axes is denoted by $O$.

The equation of the deformed fringe pattern captured by the camera, can be expressed by

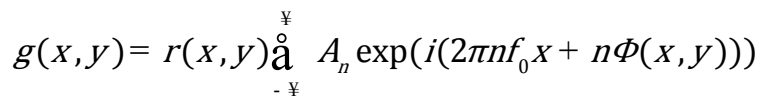

where $r(x, y)$ is the non-uniform light distribution of the diffuse object, $A_{n}$ is the weighting factor of the Fourier series, $f_{0}$ is the fundamental frequency of the observed image fringe pattern and the $\Phi(x, y)$ is phase modulation resulting from the vertical light pattern distribution.

When the inverse Fourier transform is applied to the filtered frequency, a complex signal is obtained and can be expressed by

$$
\begin{gathered}
\hat{g}(x, y)=A_{1} r(x, y) \exp \left(i 2 \pi f_{0} x+\Phi(x, y)\right) \\
\Phi(x, y)=\frac{\operatorname{Im}(\hat{g}(x, y))}{\operatorname{Re}(\hat{g}(x, y))}
\end{gathered}
$$

In this way the computed phase values are limited inside the interval $[-\pi, \pi]$ and exhibit $2 \pi$ phase jumps. To obtain the phase with continuous distribution the phase unwrapping algorithm is applied which will be described later.

The $z_{\varepsilon}$ coordinate of the measured point can be calculated by

$$
Z_{c}(x, y)=\frac{P_{y}+P_{z} \tan \left(\alpha_{i}(x, y)\right)}{V_{n}+\tan \left(\alpha_{i}(x, y)\right)}
$$

The current triangulation angle is calculated by

$$
\alpha_{i}(x, y)=\alpha_{0}+n(x, y) \Delta \alpha
$$

where $\Delta \alpha$ is an angle between light planes and the index $n(x, y)$ is

$$
n(x, y)=\frac{\Phi(x, y)}{2 \pi}
$$


The crucial step in FTP is the appropriate filtering in the frequency domain. It is highly recommended to filter out all frequencies except the fundamental one and a few of the closest neighbors. This is done using the Hann window function which is positioned around the fundamental frequency (see Fig. 3c, red ). A smaller window eliminates more noise but on the other hand filters out also the sharp edges and the discontinuities of the measured surface.

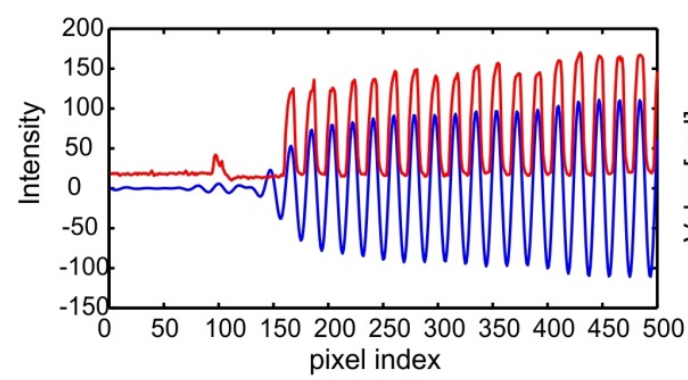

a)

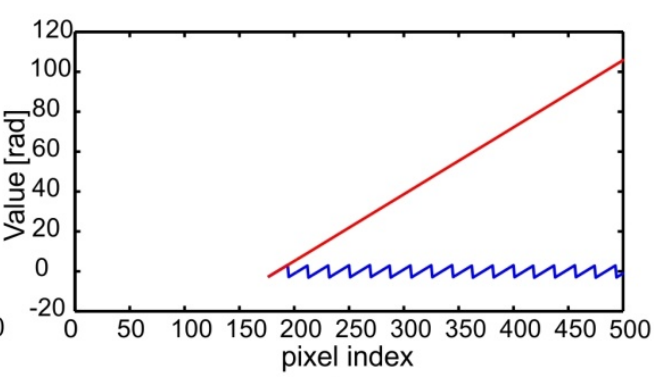

b) $\times 10^{4}$

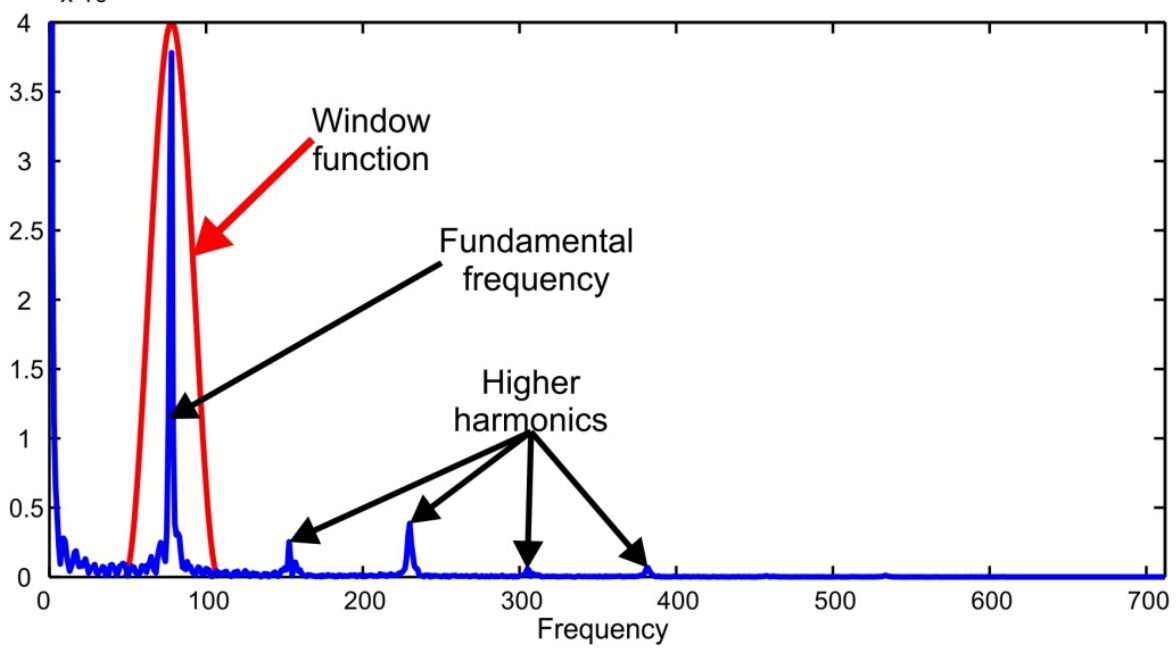

c)

Fig. 3.a) Segment of the profile of the input image (red), same segment after filtering in the frequency domain (blue). b) Wrapped phase (blue) and unwrapped phase (red). c) Frequency diagram (blue) and window function for filtering in the frequency domain (red).

The Fourier transform is performed in one direction only: perpendicular to the orientation of the stripes. Each row is transformed and filtered separately. On Fig. 3a original intensity profile (red) and intensity profile after filtering in frequency domain (blue) can be seen. When the wrapped phase image is obtained (Fig. 3b, blue curve), the 2D phase unwrapping algorithm is applied, which provides significantly better results compared to the 1D unwrapping algorithm. In our case, the algorithm presented in [10] was used. It provides a good balance between the speed of calculation and the quality of the result. The basic idea of the algorithm is that pixels with highest reliability are unwrapped first and the pixels with lowest reliability are unwrapped last in order to prevent error propagation. The reliability of the pixel is defined as the inverse of difference $D$, which is the sum of the squared values of differences of the current pixel and all of its 8 neighbors. The edge is defined as an intersection between two pixels, connected vertically or horizontally. The reliability of the edge is calculated as a sum of the reliability of both pixels. The unwrapping path is defined by considering the value of the reliability of the edges - the edges with higher reliability are resolved first. The example of the result of simple 1D unwrapping procedure is presented in Fig. 3b: the wrapped phase (blue) and the unwrapped phase (red).

The color of the surface is calculated from the captured image. The brighter parts of the image carry correct information about surface color, while the color information in the darker parts of the image is unreliable. Thus, the colors from the brighter parts of the image are interpolated across the dark parts of the image. In case of a small grating period, the error is very small.

The code for the surface reconstruction from a single image with the deformed grating pattern was developed in Visual C++ with the aid of the OpenCV library. 


\section{System calibration}

The inherent inaccuracies of the system components can influence the outcome of the measurements. In general, the inaccuracies of systematic origin (such as small differences in actual triangulation angle, lens focal length, distortion etc.), can be compensated by calibration. The presented system was calibrated using the reference surface method such as described in [14]. For this purpose an object with known geometry was manufactured with high accuracy. The reference object was measured at various distances (see Fig. 4a), from $1800 \mathrm{~mm}$ to $2100 \mathrm{~mm}$ for greater measuring range and from 900 $\mathrm{mm}$ to $1100 \mathrm{~mm}$ for smaller measuring range. The step between consecutive measurements was 25 $\mathrm{mm}$. To achieve accurate positioning the reference object was fixed on a sliding table, while the apparatus was mounted on a tripod.

About twelve calibration images were captured and imported into the software for the transformation parameter optimization. The parameters were optimized using Powell's conjugate gradient descent method [13]. The estimator of the current parameters quality was the standard deviation of the differences between the reference and measured surface. When convergence was detected, the optimization procedure stopped. After the calibration, the standard deviation of the differences between the reference and the measured surface was $1.4 \mathrm{~mm}$ for greater measuring range and 0.5 $\mathrm{mm}$ for smaller measuring range.

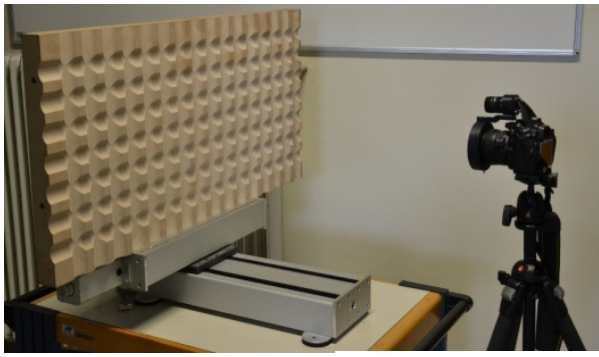

a)

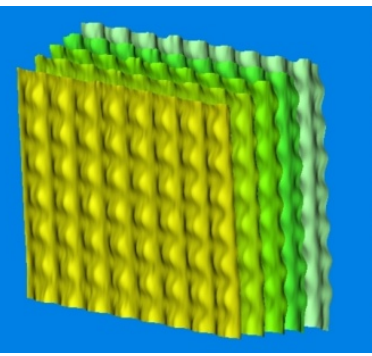

b)

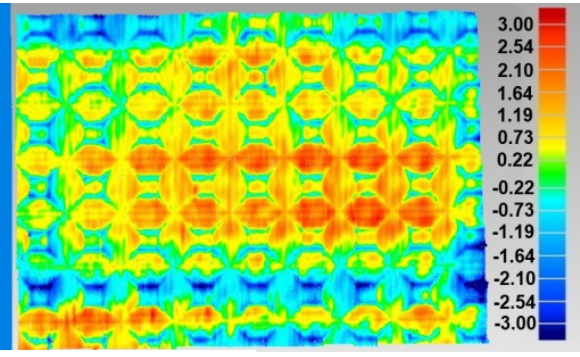

c)

Fig. 4.a) Measuring reference object. b) Measurements of reference object shape captured at different distances. c) Front view of the array of errors between ideal and measured surface after the calibration.

\section{Results and application}

The measuring system was used in different applications in cooperation with University Medical Center Ljubljana. In all cases the camera exposure time was set to 1/200 s and ISO speed between 200 and 500 and the aperture was set to $f / 11$ to obtain sufficient depth of field.

In the first application the volume of the wounds during the healing process was measured (see Fig. 5). Across the wound virtual healthy skin was approximated over the measured wound surface. The wound volume was determined as the volume between the measured surface and the virtual healthy skin. In that way the medical personnel can objectively evaluate the progress of the healing, and change the course of treatment if necessary [11].

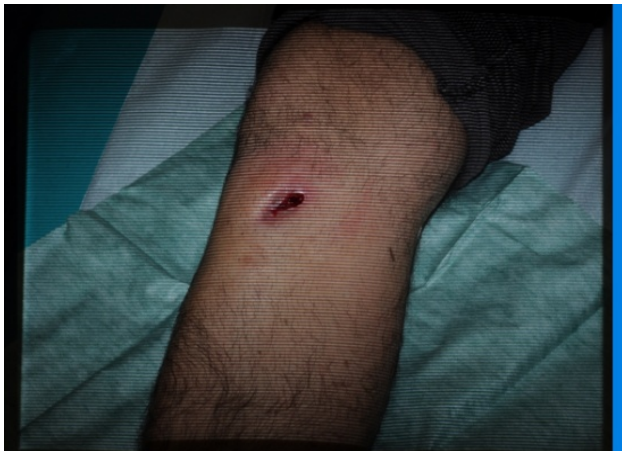

a)

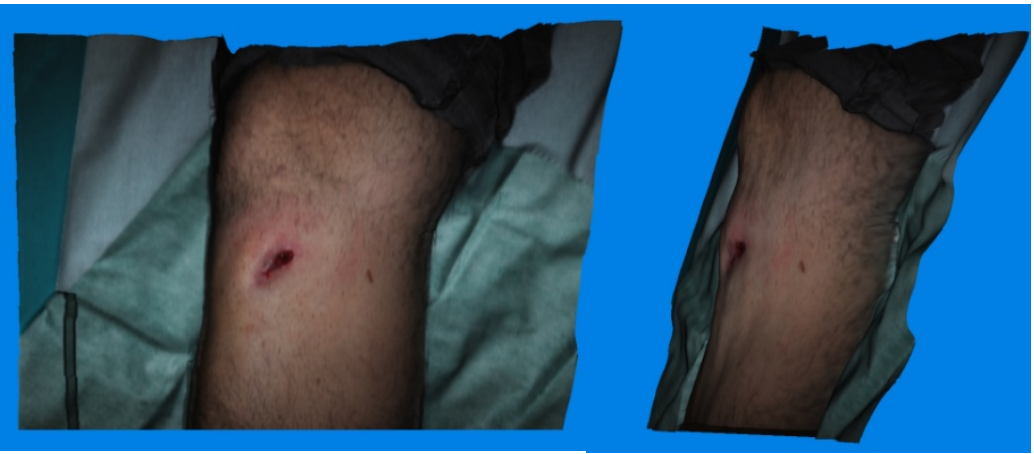

b)

Fig. 5.a) Image of the wound below the knee. b) Image of the reconstructed surface. Higher noise at some parts is caused by hair; the immediate surroundings of the wound was shaven. 
In second application the human head orientation with respect to the torso was measured. This information is needed to accurately diagnose and evaluate the course of treatment of patients with torticollis disease [15].Some example images and reconstructed surfaces with different orientations of the head can be seen in Fig. 6 .

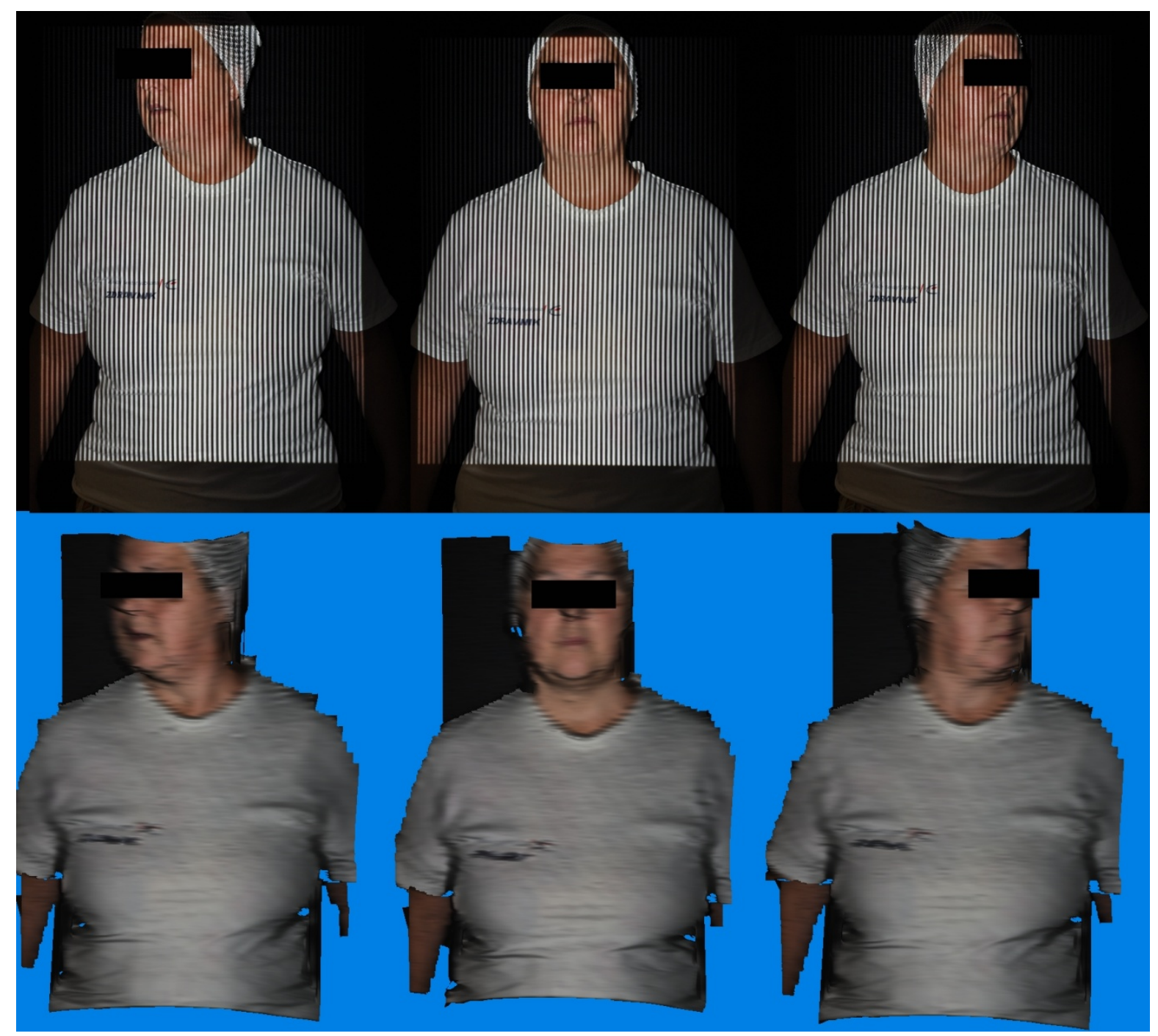

Fig. 6. Measurement of the Euler angles of the human head with respect to torso. In the upper half of the figure the captured images are presented (black background) with the corresponding reconstructed surfaces in color below each image (blue background). The noise in the reconstructed surfaces on the left side of the chest is caused by a quite dark logo on the T-shirt.

\section{Conclusion}

A handheld 3D measuring system was developed which is based on a commercial DSLR camera with a custom built structured light projector. It has measuring ranges $700 \times 520 \times 400 \mathrm{~mm}$ and $300 \times 260 \times 200$ $\mathrm{mm}$. After the calibration the standard deviation between the reference model and the measured surface was $1.4 \mathrm{~mm}$ for greater measuring range and $0.5 \mathrm{~mm}$ for smaller measuring range. For the surface reconstruction the Fourier transform profilometry was used where single image is needed. The colors of the surface can be assigned by the captured images.

To improve the measuring results on more complex surfaces, further work will concentrate on enhancing the phase unwrapping algorithm. 


\section{References}

1. http://www.creaform3d.com/en/metrology-solutions/portable-3d-scanner-handyscan-3d (accessed 2012)

2. http://www.zcorp.com/en/Products/3D-Scanners/ZScannerandtrade_800/spage.aspx (accessed 2012)

3. http://www.artec3d.com/3d_scanners/ (accessed 2012)

4. http://www.proto3000.com/3Dlaserscanning.aspx (accessed 2012)

5. http://www.nikonmetrology.com/en_EU/Products/Laser-Scanning/Handheld-scanning (accessed 2012)

6. http://polhemus.com/?page=Scanning_Fastscan (accessed 2012)

7. http://www.4ddynamics.com/3d-scanners/picoscan/ (accessed 2012)

8. Strat, A.K., Oliveira, M.M., (2003): "A point-and-shoot color 3D camera", Fourth International Conference on 3-D Digital Imaging and Modeling Proceedings, 483-490.

9. Takeda M., Mutoh K. (1983): "Fourier transform profilometry for the automatic measurement of 3-D object shapes", Appl. Opt., vol. 22, pp. 3977-3982.

10. Arevalillo Herraez M., Burton D. R., Lalor M. J., Gdeisat M. A., (2002): "A Fast two-dimensional phase unwrapping algorithm based on sorting by reliability following a non-continuous path", Appl. Opt, vol. 41, no. 35, pp. 7445-7455.

11. Kecelj-Leskovec N., Jezeršek M., Možina J., Pavlović M. D., Lunder T., (2007): "Measurement of venous leg ulcers with a laser-based threedimensional method: Comparison to computer planimetry with photography," Wound Repair and Regeneration, vol. 15, pp. 767-771.

12. Su X., Chen W., (2001): "Fourier transform profilometry: review", Opt Laser Eng; vol. 35(5), pp. 263-284.

13. Powell M. J. D., (1964): "An efficient method for finding the minimum of a function of several variables without calculating derivatives", Computer Journal, vol. 7(2), pp. 155-162.

14. Jezeršek M., Možina J., (2009): "High-speed measurement of foot shape based on multiple-laser-plane triangulation", Optical Engineering, vol. 48(11) 113604.

15. Marchetti A., Magar R., Findley L.J., Pirtošek Z., (2005): "Retrospective evaluation of the dose of Dysport and BOTOX in themanagement ofcervical dystonia and blepharospasm: the real DOSE study«, Mov. disord., vol. 20, num. 8, pp. 937-944. 